\title{
ULTRA-LOW INTENSITY PROTON BEAMS FOR RADIATION RESPONSE RELATED EXPERIMENTS AT THE U-120M CYCLOTRON
}

\author{
Tomas MatlochA ${ }^{a, b, *}$, Filip KRIZEK $^{a}$ \\ ${ }^{a}$ Nuclear Physics Institute of the CAS, p.r.i. Rez 130, 25068 Rez, Czech Republic \\ ${ }^{b}$ Czech Technical University in Prague, Faculty of Nuclear Science and Physical Engineering, Brehova 7, 11519 \\ Praha 1, Czech Republic \\ * corresponding author: matlocha@ujf.cas.cz
}

Abstract. The U-120M cyclotron at the Nuclear Physics Institute (NPI) of the Czech Academy of Sciences in Rez is used for radiation hardness tests of electronics for high-energy physics experiments. These tests are usually carried out with proton fluxes of the order of $10^{5}-10^{9}$ proton $\cdot \mathrm{cm}^{-2} \cdot \mathrm{s}^{-1}$. Some tests done for the upgrade of the Inner Tracking System of the ALICE experiment at CERN, however, required proton beam intensities several orders of magnitude lower. This paper presents a method which has been developed to achieve the proton beam flux of the order of 1 proton $\cdot \mathrm{cm}^{-2} \cdot \mathrm{s}^{-1}$. The method is mainly based on reduction of the discharge current in the cyclotron internal Penning type ion source. Influence of this new operation mode on the lifetime of ion source cathodes is discussed.

KEYWORDS: Ultra-low intensity proton flux, Penning ion source, negative hydrogen yield resonance, cyclotron irradiation facility, radiation hardness tests.

\section{INTRODUCTION}

The NPI cyclotron U-120M [1] is a four-sector isochronous cyclotron which was developed in the Joint Institute of Nuclear Research in Dubna (Russia) and installed in the NPI in 1977. Since then it has been continuously upgraded [2]. The cyclotron is tunable in a frequency range from 10 to $27 \mathrm{MHz}$ and provides proton beams with energies ranging from 6 to $37 \mathrm{MeV}$. The cyclotron can also accelerate ions with the mass to charge ratio up to $m / Q=2$ to the energies up to $10 \mathrm{MeV}$ per nucleon. An overview of the available ion beams together with their maximal energies and target beam intensities is presented in Tab. 1. The uncertainty in beam energy for protons at $35 \mathrm{MeV}$ is $\pm 0.4 \mathrm{MeV}$.

The cyclotron U-120M is mainly used for production of medical radioisotopes and for astrophysical and radio-biological experiments. In recent years, it is utilized also by experiments that test radiation hardness of various electronic components of highenergy physics detectors. The continuous upgrade of all machine technology systems allowed to reach high reliability and very good reproducibility of the beam parameters between different irradiation campaigns. This is especially important for our cooperation on the radiation hardness tests of electronics for the upgrade of the Inner Tracking System (ITS) detector of the ALICE experiment at CERN [3]. These tests require stable proton beams with fluxes below $10^{9}$ proton $\cdot \mathrm{cm}^{-2} \cdot \mathrm{s}^{-1}$. A new method to provide proton beams with such parameters had to be developed. A detailed methodology overview of the radiation hardness tests done for the ALICE ITS upgrade project

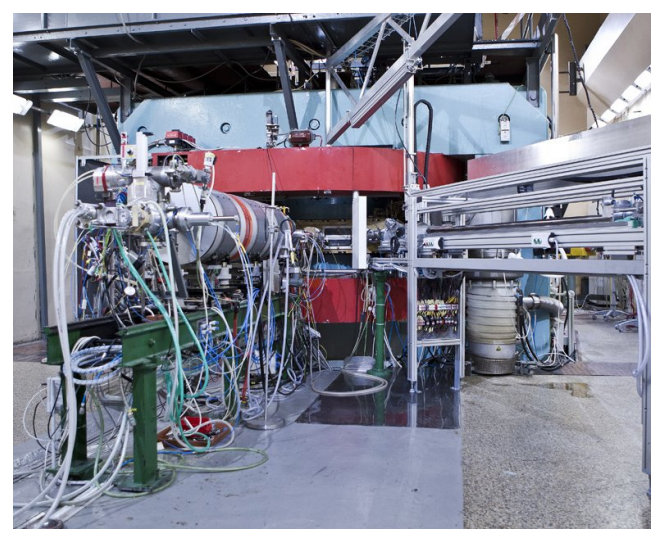

Figure 1. The cyclotron U-120M.

together with a description of the corresponding beam and dose monitoring system can be found in [4].

\section{BEAM MONITORING AND MEASUREMENT}

In the tests of radiation hardness done at the U-120M cyclotron facility, intensity of the proton beam is monitored $130 \mathrm{~cm}$ from the cyclotron negative mode beamline exit window using an ionization chamber Farmer 30010 from PTW-Freiburg [5]. The chamber is connected to a UNIDOS E Universal Dosemeter. The chamber allows to measure the proton flux in the range $10^{2}-10^{9}$ proton $\cdot \mathrm{cm}^{-2} \cdot \mathrm{s}^{-1}$. To monitor, the fluxes below $10^{4}$ proton $\cdot \mathrm{cm}^{-2} \cdot \mathrm{s}^{-1}$ a Timepix device [6 8 is used. The Timepix is a hybrid silicon pixel detector. The Timepix sensor has $256 \times 256$ pixels, an active area of $2 \mathrm{~cm}^{2}$, and a thickness of $300 \mu \mathrm{m}$, see 


\begin{tabular}{lccc}
\hline & $\mathbf{H}^{-} / \mathbf{H}^{+}$ & $\mathbf{D}^{-} / \mathbf{D}^{+}$ & ${ }^{4} \mathbf{H e}^{2+} /{ }^{3} \mathbf{H e}^{2+}$ \\
\hline Intensity internal $[\mu \mathrm{A}]$ & $-/ 200$ & $-/ 100$ & $40 / 20$ \\
\hline Intensity external $[\mu \mathrm{A}]$ & $50 / 5$ & $35 / 5$ & $5 / 2$ \\
\hline Energy $[\mathrm{MeV}]$ & $6-37$ & $11-20$ & $4-40 / 3-55$ \\
\hline
\end{tabular}

TABLE 1. Energies and intensities of the available ion beams at the U-120M cyclotron irradiation facility.

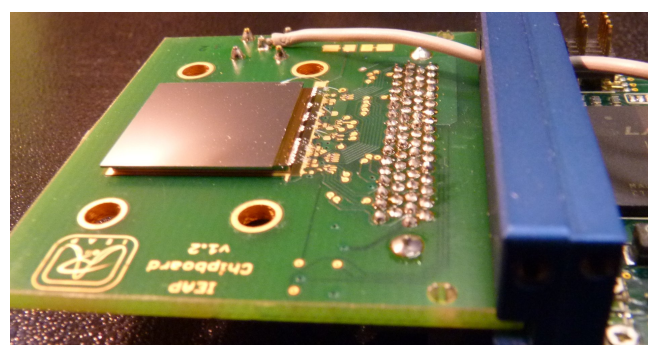

Figure 2. Detail of the Timepix sensor [6].

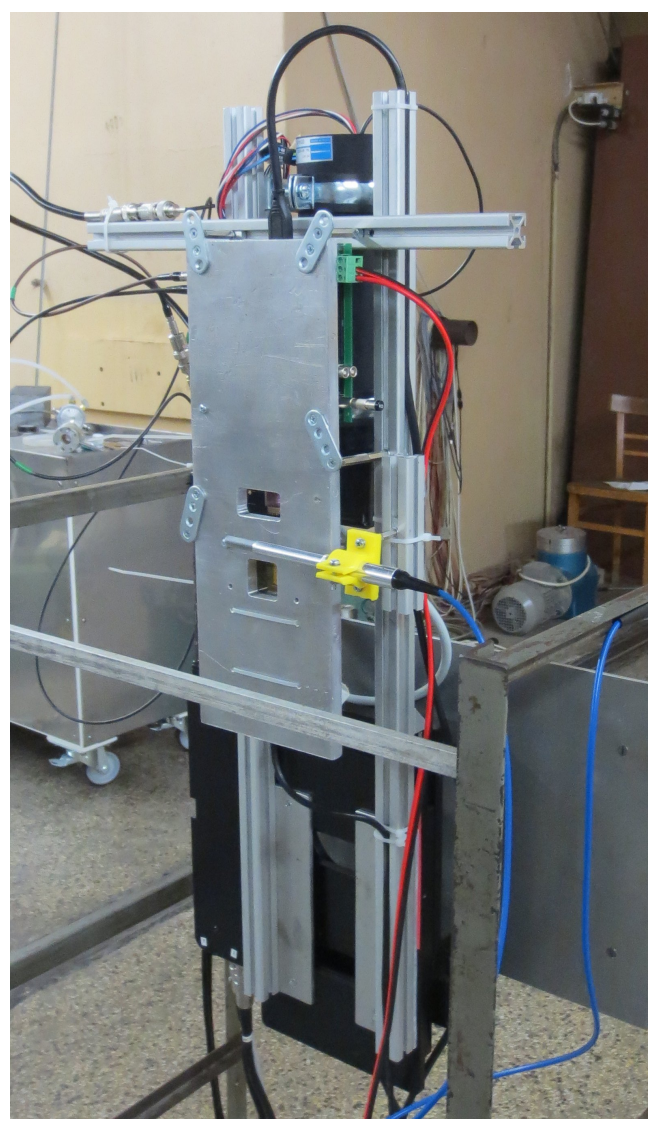

Figure 3. Irradiation setup with the Farmer PTW 30010 ionization chamber and the Timepix device.

Fig. 2. The mean energy deposited by one $30 \mathrm{MeV}$ proton in the sensor is about $1 \mathrm{MeV}$. Details about the ionization chamber calibration and the measurement setup can be found in [4]. A photo of the irradiation setup is shown in Fig. 3

\section{U-120M ION SOURCE}

The ion source is an internal cold cathode Penning type ion source (PIG). The ion source body is made of a tungsten-copper composite. The cathodes are made of tantalum due to its low sputter rates and high lifetime [9]. The tantalum cathodes yield also the highest gain of negative hydrogen ions when compared to other previously tested materials, such as molybdenum or tungsten.

The ion source works in a DC arc mode and is capable to deliver beams up to $700 \mu \mathrm{A}$ DC equivalent for $\mathrm{H}^{-}$and $2 \mathrm{~mA} \mathrm{DC}$ eq. for protons. A lifetime of the ion source for Hydrogen and Deuterium operation is several hundreds of beam hours. The maximal recorded lifetime for the $\mathrm{H}^{-}$regime is about 700 beam hours. The maintenance period is kept at 250 beam hours for reliable operation, since a failure of the ion source after about 300 beam hours can occur without any previous warning signs. This preventive maintenance thus reduces the number of unplanned cyclotron shut downs. The lifetime of the ion source for the Helium operation is $\approx 70$ beam hours for ${ }^{4} \mathrm{He}^{2+}$ ions and $\approx 30$ beam hours for ${ }^{3} \mathrm{He}^{2+}$. Regulation capabilities of the ion source are described in the next section. A scheme of the U-120M ion source is shown in Fig. 4, more details can be found in [10].

\section{BeAm intensity REgUlation}

In the negative mode of the U-120M cyclotron, the highest proton beam current that can be used to irradiate the target is about $50 \mu \mathrm{A}$. This corresponds to the total flux of $3.1 \times 10^{14}$ proton $\cdot \mathrm{s}^{-1}$. Such a high current can be used to irradiate passive devices to produce material damage but it is too high for radiation testing of electronics like silicon sensors or field programmable gate arrays. Typical fluxes that are used for the electronics tests at the U-120M cyclotron facility are in the range $10^{3}-10^{9}$ proton $\cdot \mathrm{cm}^{-2} \cdot \mathrm{s}^{-1}$.

Lowering the proton beam flux from its maximal intensity by two or three orders of magnitude can be achieved by increasing the gas pressure in the ion source and lowering of the cyclotron beam filling factor [4]. Achieving even lower fluxes requires to combine several other methods to ensure that the delivered beam is stable.

A significant difficulty for the ultra-low beam tuning process is the fact that the cyclotron internal beam current monitors have poor resolution and are not able to measure the beam current below the total 


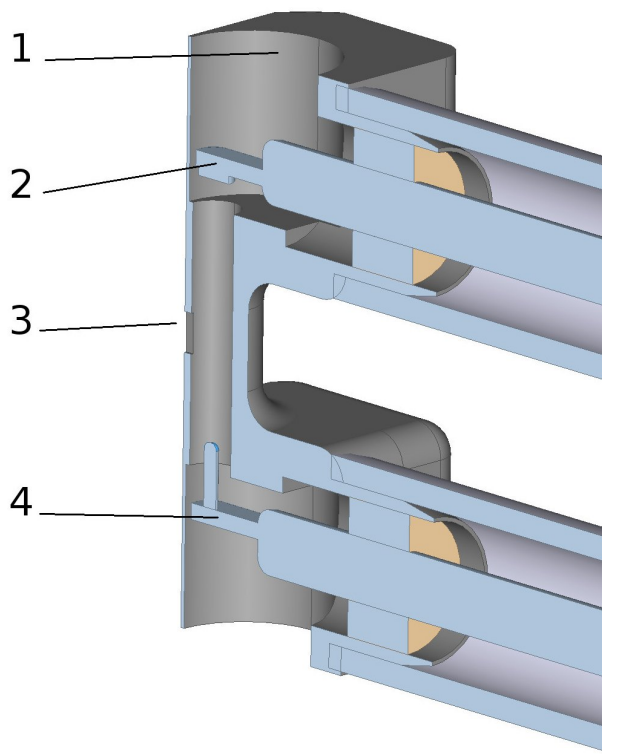

Figure 4. Scheme of the U-120M cyclotron PIG ion source. The number coding denotes the tungstencopper body of the ion source (1), the anticathode (2), the extraction slit (3), and the cathode (4).

flux of $\approx 10^{8}$ proton $\cdot \mathrm{s}^{-1}$. From this reason, the machine is first tuned properly with higher internal beam intensities for which the internal measurement is possible. Subsequently, the beam is extracted to the experimental site where the beam is focused to the desired beam spot. Finally, the beam intensity is down-regulated to the requested value based on the measurement provided by the ionization chamber.

The usual methods used for lowering the beam intensity at the U-120M cyclotron are as follows:

- reducing the filling factor of the cyclotron beam,

- worsening the vacuum quality in the cyclotron vacuum chamber,

- increasing the gas pressure in the ion source,

- shifting the horizontal position of the ion source extraction slit with respect to the slit of the puller electrode on the dee,

- lowering the arc current in the PIG ion source,

- stopping a part of the beam on an integral beam probe at the final beam orbit in the vacuum chamber,

- collimating the extracted beam on a vertical input slit of the beamline.

More detailed description of the above mentioned methods can be found below.

\subsection{U-120M RADIO FREQUENCY STRUCTURE}

The cyclotron filling factor is regulated by a pulsewidth modulation (PWM) of the main cyclotron carrier radio frequency $(\mathrm{RF}) 10-27 \mathrm{MHz}$ with a $150 \mathrm{~Hz}$ macropulsed signal. The minimal duty cycle of the modulating pulse is $3 \%$, which corresponds to about
$200 \mu \mathrm{s}$. This time is the minimal time necessary for a sufficient excitation of the cyclotron resonator. The maximal duty cycle of the macropulsed signal depends on the cyclotron RF. It is $70 \%$ for the $10 \mathrm{MHz} R F$, but only $25 \%$ for the $27 \mathrm{MHz} \mathrm{RF}$. The reason for the lower PWM range at higher RF is a sparking protection of the RF system. At present, the regulation step of the duty cycle is $1 \%$. A modification of the modulator device to allow regulation step below $0.1 \%$ is in progress.

\subsection{Regulation By VACUUm Quality AND ION SOURCE GAS}

The vacuum in the cyclotron chamber is maintained by three diffusion pumps. If some of them are switched off vacuum quality worsens. The internal beam intensity then gets lower by about one order of magnitude.

The operational margin of the beam regulation via changing the ion source hydrogen gas pressure is relatively limited. Highly non-optimal gas pressure leads to high ion source output instabilities. The ion source pressure regulation can lower the beam intensity by up to two orders of magnitude.

\subsection{ION SOURCE SHIFT}

The beam current can be effectively regulated by two or three orders of magnitude by shifting the horizontal position of the ion source extraction slit with respect to the slit of the extraction electrode on the dee, the so-called puller. The optimal position of the ion source is found by minimizing of the beam loses on the puller during the first beam orbit. When the ion source extraction slit is shifted from its optimal position, a part of the beam is stopped on the puller.

A planar view of the cyclotron central region with the ion source shifted with respect to the puller is shown in Fig. 5. However, note that stopping a very intense beam on the puller warms up the electrode. Consequently, there can be thermally stimulated emission of electrons to the cyclotron vacuum chamber which leads to sparking. Thus for stable operation, it is necessary to pre-regulate the beam intensity extracted by the puller from the ion source.

\subsection{ION SOURCE DISCHARGE CURRENT REGULATION}

In routine operation, the arc current is kept near $1 \mathrm{~A}$ which ensures stable operation. When the arc current is below $0.5 \mathrm{~A}$ and over $1.4 \mathrm{~A}$ the ion source can became unstable, especially in the second half of its cathode lifetime. In order to find a way how to reduce the proton flux, the ion source was studied at the test bench with the goal to investigate whether the proton flux can be regulated by the ion source discharge current. In these measurements, the ion source was operated in a pulse mode. The voltage pulses had a length of a few tens of millisecond. It was observed that the yield of negative hydrogen ions is maximal at the beginning of the pulse. A few milliseconds after 


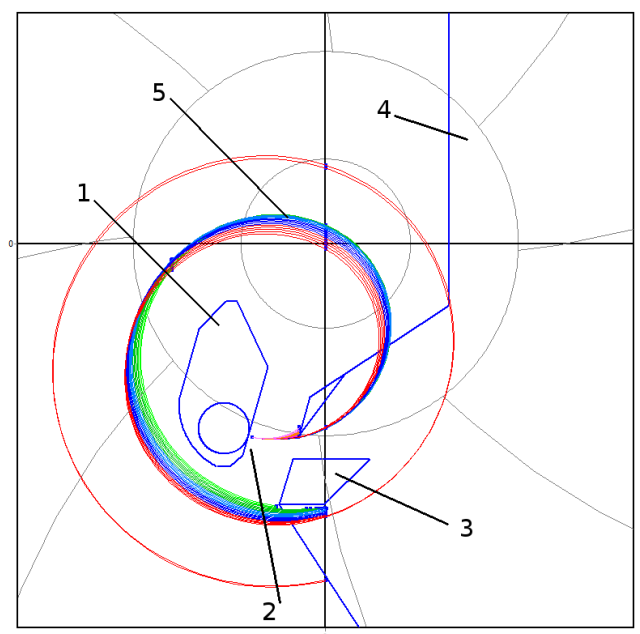

Figure 5. Planar view showing the shifted extraction slit (2) of the ion source (1) with respect to the puller (3) on the dee (4). A part of the beam (5) is stopped on the puller during the first orbit. Simulated in Durycnm4 [11.

the ignition, the intensity of ions decreased as the cathode warmed up. However, operation of the ion source in the pulse mode turned out to be problematic, because the ignition process in the PIG ion source was unstable. With $10 \mathrm{~ms}$ long pulses and a repetition rate up to $10 \mathrm{~Hz}$, it was noted that the ignition got delayed with respect to the beginning of the high voltage pulse. A part of the pulses did not start properly, and the moment of the ignition of the ion source discharge was randomly floating in the first millisecond. The problem with the delayed ignition was not solved even when rising the ignition voltage up to $3000 \mathrm{~V}$ from its nominal value $1500-2000 \mathrm{~V}$. In addition, a part of the pulses, which started properly at the beginning of the high voltage pulse, exhibited significant ringing, i.e. the ion source discharge current highly oscillated at the beginning of the pulse until the arc got stabilized. Both issues were strongly suppressed when we introduced a small, residual constant discharge current of $5 \mathrm{~mA}$. The pulse operation of our PIG ion source then became stable with rectangular discharge current pulses. A deeper investigation of the ion source parameters at the test bench showed that the ion source discharge current in the milliampere region is stable, if the cathodes are new or in a very good condition. The ion source pulsed mode was finally not utilized for the cyclotron operation, but the regime with the small residual currents has found its use in experiments that require a low flux of protons.

\subsubsection{The MINIMAL ION SOURCE DISCHARGE CURRENT}

The minimal stable current of the PIG ion source discharge measured at the test bench was about $2 \mathrm{~mA}$. For lower discharge currents, the arc switched off. When the measurement of the lowest possible discharge current was repeated at the cyclotron with

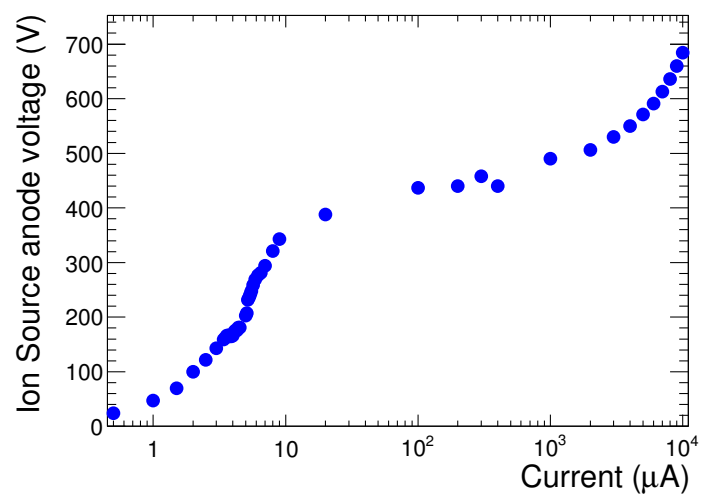

Figure 6. Current-voltage characteristic of the ion source for small discharge currents.

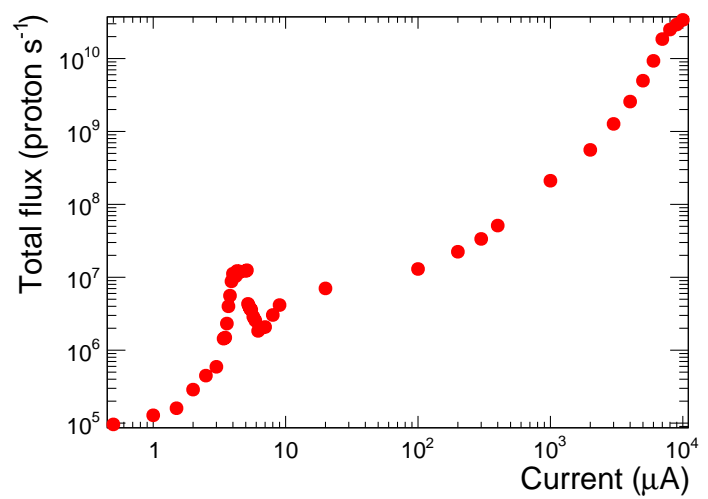

Figure 7. Total cyclotron flux as a function of the ion source arc current. Figure taken from [12].

RF being switched on, it was observed that the ion source discharge current did not have any practical lower limit. Let us point out that usually, the PIG ion source needs some minimal voltage, typically several hundreds volts, to ignite. In the measurement done at the cyclotron, the discharge current started to increase almost linearly as the anode voltage exceeded $\approx 10 \mathrm{~V}$, see the measured current-voltage characteristic in Fig. 6. In the range $0-3.5 \mu \mathrm{A}$, the ion source behaved as a pure resistive element.

Figure 7 shows the measured total proton flux as a function of the ion source discharge current in the range $1-1000 \mu \mathrm{A}$. When switching the ion source off, the cyclotron still provided a residual total proton flux of about $5 \times 10^{4}$ proton $\cdot \mathrm{s}^{-1}$, see Fig. 8 This residual proton flux can be explained based on the following considerations. The distance between the ion source and the puller is about $3 \mathrm{~mm}$ and the amplitude of $\mathrm{RF}$ voltage is $34 \mathrm{kV}$. As a result there is a strong electric field (about $10 \mathrm{MV} / \mathrm{m}$ ) between the puller electrode of the dee and the grounded ion source. This strong electric field stimulates an emission of electrons from the puller surface. The electrons get accelerated and ionize the neutral gas leaking from the ion source. This effect probably causes the primary ionization. 


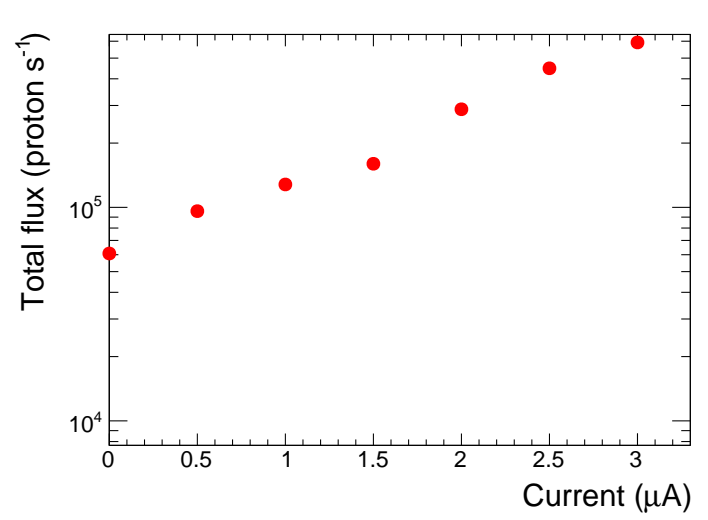

Figure 8. Total proton flux as a function of the ion source discharge current. The zero current value correspods to the ion source switched off. Figure taken from 12 .

The produced space charge partly leaks back into the ion source and causes its almost linear behavior in the range of anode current $0-10 \mu \mathrm{A}$. This operation mode of the ion source was successfully utilized for the low proton flux beam experiments at the NPI irradiation facility.

The flux of $\mathrm{H}^{-}$as a function of the ion source discharge current exhibits also a pronounced narrow resonant structure at $3-5 \mu \mathrm{A}$, see Fig. 7. The resonance is clearly visible also in Fig. 9 which shows the ion source efficiency versus the ion source current. The ion source efficiency is defined as the number of $\mathrm{H}^{-}$ions produced per unit ion source discharge power. This resonance occurs in all conducted experiments with various magnetic field and different ion source gas pressure. The amplitude of the resonance depends on the ion source gas pressure and its position slightly shifts when the cyclotron magnetic field is changed. For the experiments which require a low intensity proton beam, it is necessary to avoid operation in this resonance region, as there the flux varies considerably with any subtle change of the ion source discharge current. To our knowledge, the observed resonance is not described in the literature and possibly may find use in the fields where an energetic efficiency is important, e.g. in ion thrusters.

\subsection{The CATHOdes EMBRITTLEMENT}

Our experience gained from the operation of the ion source in the regime with very low discharge currents shows that the cathodes become very fragile and after several tens of beam hours fall apart. This hydrogen embrittlement phenomenon is well known [13]. The low discharge current regime, however accelerates this process significantly. The cathodes get much more fragile when running 50 hours with $500 \mu \mathrm{A}$ ion source discharge current than after running several hundreds beam hours with $1 \mathrm{~A}$. The lower cathodes temperature caused by the low discharge current, probably do not allow the hydrogen to be properly annealed. Until the

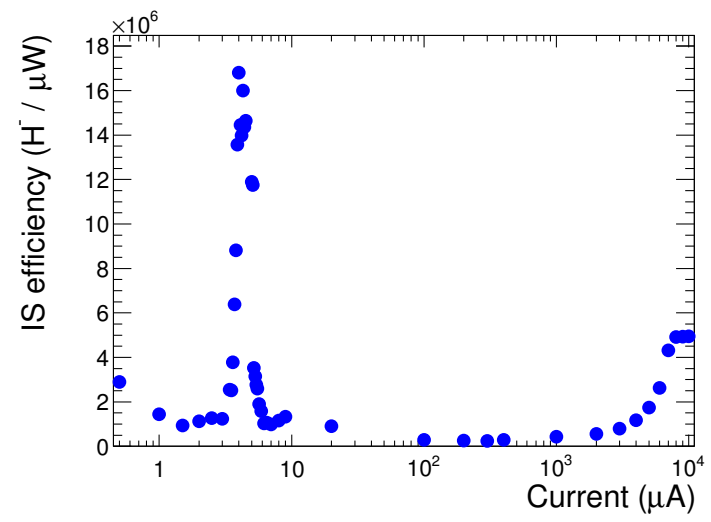

Figure 9. Negative hydrogen yield efficiency versus ion source discharge current.

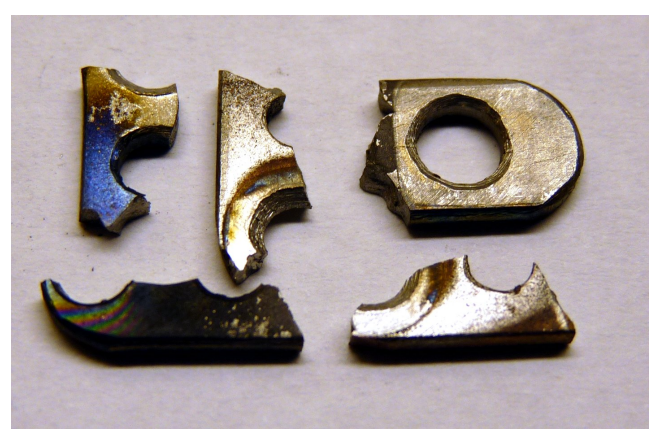

Figure 10. Disintegrated cathodes after embrittlement by a hydrogen diffusion into tantalum.

embrittlement issue will be solved, the cathodes have to be replaced after several tens of beam hours, to prevent that they fall apart and cause the cyclotron unplanned shutdown. A photo of the cathodes that got broken after 80 beam hours is shown in Fig. 10

\section{The Minimal BEAM INTENSITY}

With the current experience, the proton fluxes in the range $10^{3}-10^{9}$ proton $\cdot \mathrm{cm}^{-2} \cdot \mathrm{s}^{-1}$ can be achieved in relatively short time $(\approx 5 \mathrm{~min})$ using just the basic tune of the cyclotron and the beamline elements following the settings from previous runs. Then the output proton flux can be easily controlled by the ion source discharge current. On the other hand, tuning of the fluxes below $10^{3}$ proton $\cdot \mathrm{cm}^{-2} \cdot \mathrm{s}^{-1}$ requires more time $(\approx 30 \mathrm{~min})$. It is necessary to combine all of the above mentioned intensity lowering techniques with a special care given to the stability of the beam.

Figure 11 illustrates density of protons on the Timepix sensor for fluxes in the range $1-10^{3}$ proton . $\mathrm{cm}^{-2} \cdot \mathrm{s}^{-1}$. After a proper tune, the flux can be continuously regulated over the whole range only by the ion source discharge current. The minimal possible flux reachable at our irradiation facility was estimated to be about 0.05 proton $\cdot \mathrm{cm}^{-2} \cdot \mathrm{s}^{-1}$. The stability of intensity for the fluxes over $10^{3}$ proton $\cdot \mathrm{cm}^{-2} \cdot \mathrm{s}^{-1}$ is better than $10 \%$ 4.

Using the proper Timepix settings, the Timepix 

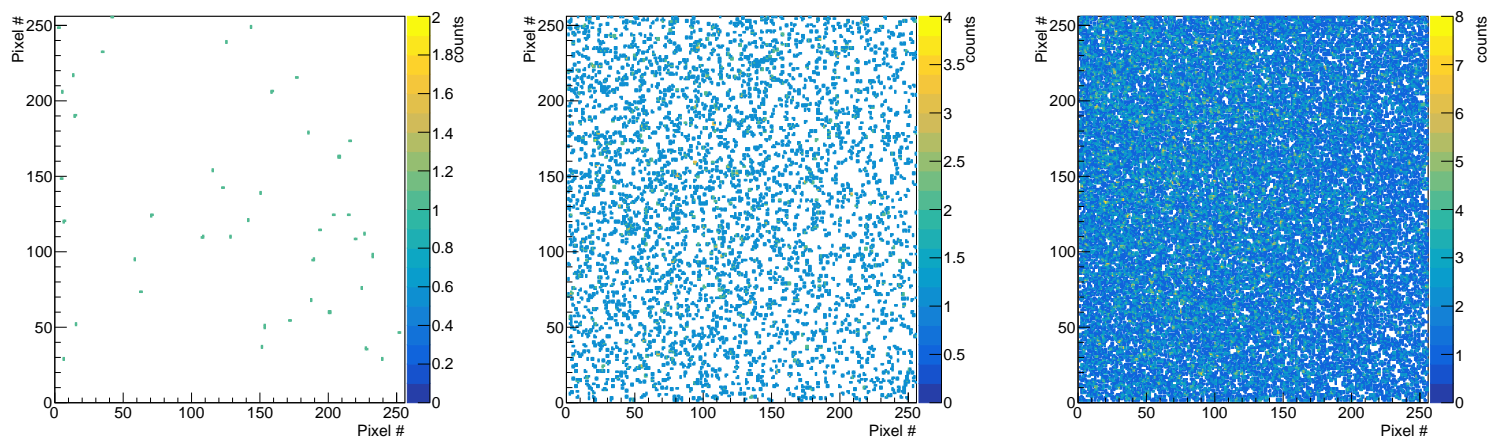

Figure 11. The ultra-low proton fluxes at the U-120M cyclotron for various ion source discharge currents. Timepix collection time 30 seconds, bias $5 \mathrm{~V}$. Left: The ion source off, flux $\sim 0.7$ proton $\cdot \mathrm{cm}^{-2} \cdot \mathrm{s}^{-1}$. Middle: The discharge current $60 \mu \mathrm{A}$, flux $\sim 60$ proton $\cdot \mathrm{cm}^{-2} \cdot \mathrm{s}^{-1}$. Right: The discharge current $200 \mu \mathrm{A}$, flux $\sim 420$ proton $\cdot \mathrm{cm}^{-2} \cdot \mathrm{s}^{-1}$. Figure taken from [12].

detector counts individual protons with $100 \%$ efficiency. The measurement of the average proton flux with Timepix assumes that the $1 \mathrm{~ms}$ long time frames are distributed uniformly in time. The uncertainty in the proton flux is then given by the uncertainty in proton counting. Since proton cluster merging is small for ultra low fluxes the estimated uncertainty is better than $5 \%$.

\section{CONCLUSiOn}

The U-120M cyclotron irradiation facility is able to provide ultra-low intensity proton beams which are suitable for experiments that study response of a detector to individual particles. The minimal reachable intensity of the proton flux that was reached was well below 1 proton $\cdot \mathrm{cm}^{-2} \cdot \mathrm{s}^{-1}$. The proton flux can be continuously regulated from $10^{-1}$ to $10^{14}$ proton $\cdot \mathrm{cm}^{-2} \cdot \mathrm{s}^{-1}$. Good stability of the beam parameters is guaranteed for fluxes greater than $\approx 10^{3} \operatorname{proton} \cdot \mathrm{cm}^{-2} \cdot \mathrm{s}^{-1}$.

\section{ACKNOWLEDGEMENT}

The authors thank the Institute of Experimental and Applied Physics, Czech Technical University in Prague for providing the Timepix device. This work was supported by the Ministry of Education, Youth and Sports of the Czech Republic projects LM2015056 (CANAM), LM2015058 and LTT17018.

\section{REFERENCES}

[1] Nuclear Physics Institute of the CAS, Czech Republic. Isochronous cyclotron U-120M.

http://accs.ujf.cas.cz

[2] M. Cihak, J. Lacman, J. Stursa . New control system for the isochronous cyclotron $\mathrm{u}-120 \mathrm{~m}$. Proceedings of the 15th International Conference on Cyclotrons and their Applications 1998. http://accelconf.web.cern. ch/AccelConf/c98/papers/h-05.pdf

[3] B. Abelev ( The ALICE Collaboration). Upgrade of the alice experiment: Letter of intent. Journal of Physics G: Nuclear and Particle Physics 41, 2014. DOI:10.1088/0954-3899/41/8/087001
[4] F. Krizek et al. . Cyclotron based irradiation setup for alice inner silicon tracker upgrade - preprint. Nuclear Inst and Methods in Physics Research, A, NIMA-D-17-00930 2017.

[5] PTW Freiburg, Germany. PTW Farmer Ionization Chambers. http://www.ptw.de/farmer_chambers0.html

[6] X. Llopart, R. Ballabriga, M. Campbell, L. Tlustos, W. Wong . Timepix,a 65k programmable pixel readout chip for arrival time, energy and/or photon counting measurements. Nucl Instrum Methods Phys Res A 581:485-494, 2007. DOI:10.1016/j.nima.2007.08.079.

[7] T. Holy, J. Jakubek, S. Pospisil, J. Uher, D. Vavrik, Z. Vykydal . Data acquisition and processing software package for medipix2. Nucl Instrum Methods Phys Res A 563:254-258, 2006. DOI:10.1016/j.nima.2006.01.122.

[8] Z. Vykydal, J. Jakubek, S. Pospisil. Usb interface for medipix2 pixel device enabling energy and position-sensitive detection of heavy charged particles. Nucl Instrum Methods Phys Res A 563:112-115, 2006. DOI:10.1016/j.nima.2006.01.114

[9] S. Busold, H. Rocken, J. M. Schippers, A. S. Partowidjojo . Recent ion source developments for varians probeam cyclotron. Proceedings Cyclotrons 2016 pp. 316-318, 2016. DOI:10.18429/JACoW-Cyclotrons2016-THP06

[10] T.Matlocha. Ion source for a single particle accelerator. Acta Polytechnica CTU Proceedings 4:50-55, 2016. https://ojs.cvut.cz/ojs/index.php/APP/article/ view/3743/3808 DOI:10.14311/AP.2016.4.0050

[11] Milan Cihak, Jan Stursa, Ondrej Lebeda. Beam dynamic simulation in the isochronous cyclotron u-120m. Cyclotrons and Their Applications, Eighteenth International Conference pp. 385-387, 2007. http://accelconf.web.cern.ch/AccelConf/c07/ PAPERS/385.pdf

[12] T.Matlocha. Modification of a classical penning ion source operating mode for sub-femtoampere beams at the $\mathrm{u}-120 \mathrm{~m}$ cyclotron - in review. AIP Proceedings of 17th International Conference on Ion Source 2017.

[13] A. Baumel, P. Drodten, E. Heitz, R. Bender. Tantalum, niobium and their alloys - Corrosion Handbook, 2008. DOI:10.1002/9783527610433.chb02140 